\title{
El aula abierta en espacios naturales: Una experiencia en el Bosque Ramón Álvarez
}

\section{Open Classroom in Natural Spaces: an Experience in the Ramón Álvarez Forest}

\author{
Ana Isabel Pereira Pérez ${ }^{1}$ \\ Sede de Guanacaste \\ Universidad de Costa Rica \\ Guanacaste, Costa Rica \\ pereiraanaisabel@yahoo.com \\ Nuria Mairena Rodríguez ${ }^{2}$ \\ Sede de Guanacaste \\ Universidad de Costa Rica \\ Guanacaste, Costa Rica \\ nurimai@gmail.com
}

Recibido 07 de noviembre de $2010 \bullet$ Aceptado 09 de marzo de 2011

Resumen. El artículo presenta la experiencia de acercar los espacios naturales a la realidad educativa, como una estrategia para la formación de valores en educación ambiental y conservación. La misma constituye una experiencia de aula abierta en espacios naturales, ejecutada en el Bosque Ramón Álvarez y desarrollada por docentes universitarios y estudiantes de la Sede Guanacaste, de la Universidad de Costa Rica. En dicha experiencia, se han integrado docentes, niños y niñas de instituciones educativas de la región.

Palabras claves. Aula abierta, educación ambiental, áreas naturales, instituciones educativas, bosque conservación.

Abstract. This paper shows the experience of bringing the natural spaces closer to the educational reality as a strategy to teach values in environmental education and conservation. This open classroom activity was developed by professors and students of the Chorotega regional center, of the Universidad de Costa Rica, in the Ramón Álvarez forest. Teachers and children from the different educational institutions of this region have participated in the project.

Keywords. Open classroom, environmental education, natural areas, educational institutions, forest conservation.

\footnotetext{
Máster en Ornitología de la Universidad de Costa Rica. Bachiller en Biología de la Universidad de Costa Rica. Coordinadora de la Iniciativa Cultura Ambiental en Acción, UCR-CONARE-Región Chorotega. Profesora universitaria en la carrera de Biología de la Universidad de Costa Rica, en la sede Central y de la carrera de Turismo Ecológico en la Sede de Guanacaste. Experiencia como investigadora en el área de la Ornitología. Experiencia en asesorías de impacto ambiental. Posee artículos publicados en las áreas de ecología de alimentación de aves limícolas, dispersión de semillas por aves y camuflaje de nidos de aves.

2 Graduada en la Universidad de Costa Rica como Magistra en Educación, énfasis en Adminstración Educativa, Licenciada en Currículo y Bachiller en Educación Preescolar. Docente universitaria para las carreras de Educación Preescolar, Primaria, Preescolar y Primaria con énfasis en Inglés. Coordinadora de las carreras de Bachillerato y Licenciatura en Educación Preescolar en la Sede de Guanacaste, Universidad de Costa Rica. Directora y lectora en tesis de grado en la Universidad de Costa Rica en Educación. Responsable de proyectos de investigación y acción social. Tutora del Posgrado en Gestión y Liderazgo de la Universidad Nacional, Región Chorotega. Responsable y colaboradora en iniciativas CONARE, CRI Chorotega Cultura Ambiental en Acción y Centro de Intervención Pedagógica: Dejando Huellas.
} 


\section{Introducción}

Este artículo presenta los resultados de una experiencia desarrollada por docentes de la Sede Guanacaste, Universidad de Costa Rica, institución de educación superior estatal, cuya sede central se ubica en la provincia de San José y cuenta con 6 Sedes regionales en diferentes puntos del país. A través de esta experiencia, con ayuda de sus docentes, se ha buscado acercar al Bosque Ramón Álvarez, a los niños y niñas de instituciones educativas de la región.

La estrategia de aula abierta en espacios naturales se ha ejecutado en una fase piloto, con el afán, no solo de que los estudiantes y las estudiantes de instituciones de la región conozcan parte de la riqueza natural del país, sino también, para que, desde pequeños, desarrollen valores conservacionistas y así, poco a poco, vayan generando un efecto multiplicador en las familias y en las próximas generaciones. Se han incorporado, también, en esta fase piloto, al estudiantado de la Carrera de Turismo Ecológico y de Educación Primaria y Preescolar, impartidas en la Sede Guanacaste.

\section{Contextualización teórica}

Costa Rica ha sobresalido a nivel mundial por la riqueza biológica que alberga, la cual constituye cerca del 5\% de la biota del planeta, con tan solo el 0,03\% de la extensión global (Wainwright, 2002; Zamora, Jiménez y Poveda, 2002). Esta riqueza biológica se debe a diversos factores, como son la posición que ocupa nuestro país, entre dos grandes masas continentales, cada una de las cuales aporta parte de su riqueza biológica a nuestro territorio; y entre dos océanos, los cuales provee gran riqueza de especies marinas y moderan en parte nuestro clima (Coen, 1991). La riqueza topográfica también ejerce un efecto positivo, incrementando la diversidad ambiental y climática que repercute en la cantidad de especies que un ambiente puede albergar (Leenders, 2001; Zuchowski, 2005).

$\mathrm{Su}$ diversidad, también es rica a nivel de ecosistemas, se encuentran en nuestro territorio, importantes áreas de bosque tropical húmedo y muy húmedo, sistemas de humedales de importancia mundial, bosques nubosos, páramos y bosque seco tropical restringido al pacífico norte de Costa Rica, entre otros (Hartshorn, 1991; Valerio, 1991).

Esta riqueza natural, está protegida en parte, por diversas leyes que se han establecido desde 1969 en adelante, como son: Ley Forestal en 1969, Ley de la Conservación de Vida Silvestre en 1970 y Ley de Biodiversidad en 1981. Estas favorecieron la creación de todo un sistema de áreas protegidas y, a partir de 1989, se crea el Sistema Nacional de Áreas de Conservación, con la intención de mejorar el manejo y conservación de las áreas silvestres existentes (Barrantes, 2000; García, 2002; Meza, 2001). En total, estas áreas comprenden el 25\% del territorio nacional y su sistema de protección permite la visitación y, por tanto, el disfrute de su biodiversidad y bellezas escénicas, así como la ejecución de estudios biológicos de interés internacional (Calderón, 2005; Quesada, 2008).

Nuestras áreas boscosas y demás ecosistemas naturales brindan diversos servicios ambientales, que permiten que los seres humanos tengamos una buena calidad de vida. Dentro de estos servicios, podemos citar el control de erosión por parte de nuestros bosques; la calidad del aire, pues a través de procesos naturales como la fotosíntesis de las plantas, se remueve el $\mathrm{CO}_{2}$, uno de los gases invernadero más importante, y se libera el oxígeno fundamental para la gran mayoría de los seres vivos; regulación del clima; regulación y purificación del agua; regulación de enfermedades humanas; control biológico de plagas, y la mitigación de riesgos, entre otros (Moreno, 2005). 
Más allá de la rentabilidad, de su capacidad para la producción de madera o de la conservación de una de las concentraciones de biodiversidad más grandes del mundo, los ecosistemas boscosos son indispensables para la sobrevivencia y la buena calidad de vida de las comunidades, tanto rurales como urbanas. (Baltodano, 2008, p. 9)

La provincia de Guanacaste se caracteriza por poseer una de las zonas de vida más frágil y menos protegida del mundo: el bosque seco (Sánchez-Azofeifa et al., 2003). Debido a las condiciones climáticas que posee este ecosistema, ha sido fuertemente explotado para la ganadería (Ewel 1999), lo cual ha generado una transformación de los ecosistemas naturales a grandes extensiones de pastizales. Según indican Davis y Holmgren (2000), entre 1950 y 1984 el área dedicada a estas actividades pasó de un 12,2\% a un 32,3\% de la superficie total del país, debido al creciente mercado internacional de carne. Sin embargo, estas actividades no favorecieron a los campesinos de la región, quienes optaron por talar sus propiedades, para convertirlas en pastizales y luego venderlas a inversionistas con suficiente solvencia económica para poder convertirlas en fincas ganaderas.

La deforestación, asociada a la creciente expansión urbana y al crecimiento poblacional, ha impactado las cuencas hidrográficas y, por tanto, la disponibilidad de agua potable para las comunidades (Merayo, 2004; Ramírez 2010). La eliminación de la cobertura vegetal, en las partes altas de las cuencas, ha generado serias alteraciones del régimen hídrico, dando pie a problemas en la disponibilidad de agua potable (Hernández y Zúñiga, 2005). El agua es un recurso fundamental, no solo para las actividades agrícolas, industriales y comunales, sino también, para el equilibrio y sobrevivencia de los ecosistemas (Castro, Monge, Rocha y Rodríguez, 2004).

El Bosque Ramón Álvarez, es un espacio natural, administrado por la Universidad de Costa Rica, ubicado en Barrio Limón, Santa Cruz, Guanacaste. Posee una extensión de 11 Has de bosque secundario, que conserva especies propias de la región, como el guanacaste, ceibo, espavel, ron ron y surá, entre otros, que le dan gran valor al fragmento, pues algunos de ellos, son centenarios.

La zona corresponde a un área transicional entre el bosque seco y húmedo, con precipitaciones promedio de $1700 \mathrm{~mm}$ y temperatura promedio de $27.7^{\circ} \mathrm{C}$. Se encuentra a escasos $200 \mathrm{~m}$ del río Diriá, por lo que algunas de las especies presentes en el bosque se movilizan entre este y la vegetación colindante con el río.

En un sector del bosque fue construida un aula de 15 metros de largo por 10 de ancho, que posee un corredor amplio, servicios sanitarios, electricidad y agua potable, por lo que tiene las condiciones básicas para realizar actividades educativas (Fig. 1).

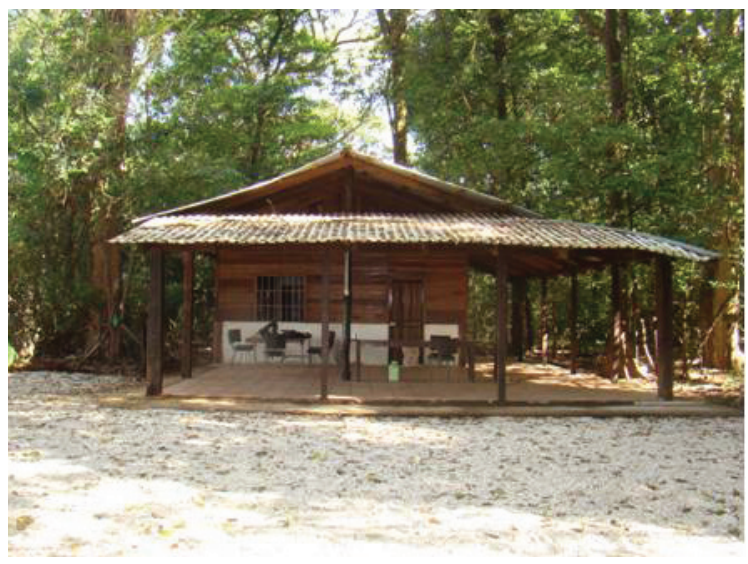

Figura 1. Instalaciones presentes en el Bosque Ramón Alvarez, donde se llevan a cabo las actividades iniciales en los recorridos de historia natural. 


\section{Aula abierta en espacios naturales}

Como parte de los factores que motivaron el desarrollo de la iniciativa de utilizar el bosque como una aula abierta, se pueden destacar, entre otros: la escasa educación sobre problemas ambientales en la Región Chorotega; los problemas de contaminación de ríos y tierras, que ponen en peligro a las comunidades; la falta de capacitación en el manejo sostenible de los recursos naturales y su renovación; así como la no organización de las comunidades para realizar actividades ambientales y culturales para su beneficio.

La conceptualización y aplicación piloto de una experiencia mediante un aula abierta en espacios naturales se ha propuesto, entre otros aspectos:

a) Realizar un estudio de la situación y análisis de las condiciones en las comunidades educativas o regiones participantes.

b) Identificar los problemas ecológicos, ambientales y forestales que ocasionan gran impacto al ambiente y a la sociedad.

c) Emplear y abordar la iniciativa de educación ambiental desde una perspectiva holística y respetando la diversidad sociocultural de las instituciones participantes. Entender que cada persona es miembro de una comunidad de partes interdependientes.

d) Incluir actividades de sensibilización ambiental, concientización ecológica, valoración del ambiente y legislación ambiental, pero sobre todo actividades prácticas.

e) Educar para la responsabilidad, de manera que las personas se transformen en consumidores sustentables.

f) Crear una conciencia ambiental en donde cada ser humano es parte de la Naturaleza. Entender que la comunidad humana incluye también el suelo, el aire, el agua, las plantas y los animales.

g) Despertar valores de solidaridad y respeto con el ambiente.

h) Mantener una meditación crítica acerca de las consecuencias de nuestras acciones y nuestros estilos de vida.

i) Declarar que toda especie tiene derecho a la supervivencia continuada en el ambiente en que vive.

j) Concientizar en que nuestro bienestar actual no debe tener preferencia sobre el de las generaciones futuras.

Estos principios brindan el marco de partida para proponer un cambio de valores, que dé sus frutos no solo en la formación de las nuevas generaciones, sino que también repercuta en el fortalecimiento de valores, actitudes y comportamientos de la comunidad que circunda el Bosque Ramón Álvarez. Así, este se constituye en una alternativa para crear, en sus visitantes, una consciencia ambientalista, al propiciar actividades permanentes que los seduzcan para que promuevan actitudes y acciones concretas hacia la conservación de los espacios naturales.

Aunque la educación ambiental se encuentra incorporada como contenido dentro de los planes curriculares del sistema educativo costarricense, en muchos casos, se desarrolla, únicamente, proporcionando la información a la población estudiantil, mediante estrategias metodológicas participativas, tales como: confección de carteles, murales, realización de diferentes concursos; pero, en la mayoría de los casos, no se da seguimiento a las acciones que generen un verdadero cambio de actitud, ni se suelen promover prácticas constructivistas para integrar y ser partícipe de acciones como reforestación, conservación y manejo de los recursos naturales. 
A raíz de la necesidad de impactar en la población, con respecto a la forma tradicional de orientar la educación ambiental, surge el proyecto "Aula abierta" como una estrategia metodológica de flexibilización curricular participativa, dirigida hacia una población diversa, con características particulares, pero con un objetivo común: adquirir responsabilidad ambiental. Según Gutiérrez (2006):

La flexibilidad curricular, en su acepción más amplia, supone la introducción de un modelo organizativo abierto, dinámico y polivalente que permita transformar las estructuras académicas rígidas y producir nuevas formas de organización mediadas por la interdisciplinariedad y el trabajo integrado, más autónomo y orientado al aprendizaje de los estudiantes. (p. 12)

El proceso mediante el cual se desarrolla el proyecto de Aula abierta está a cargo de un equipo transdisciplinario, conformado por profesionales en Educación, Biología, Turismo Ecológico, Artes Dramáticas, Ciencias, Idiomas, Historia, entre otras. Para garantizar la consecución de los objetivos previstos, el aporte de cada profesional se desarrolla en forma integrada.

Un aula abierta en el Bosque Ramón Álvarez se traduciría en ese espacio educativo en donde el discente niño, niña, docente, joven, adulto o adulto mayor interactúen, observen, manipulen; reciban charlas, cine foros; realicen recorridos y, lo más importante, entren en contacto directo con el ambiente natural y las posibilidades de contacto con seres vivos que se reproduzcan en el Bosque.

Las actividades del aula abierta son fortalecidas en las comunidades educativas, mediante estrategias metodológicas dirigidas a esta población, según las necesidades detectadas en ellas. Por otra parte, el aula abierta, también, transfiere sus acciones directamente a las instituciones educativas para complementar las celebraciones relacionadas con temas ambientales, establecidas dentro del calendario escolar, tales como: Día Mundial del Agua, Día del Ambiente, Semana de los Recursos Naturales, Día de la Tierra y Día del Árbol, entre otros.

Con la experiencia de Aula abierta en espacios naturales, se ha pretendido fomentar el interés por la conservación y preservación de los ambientes naturales presentes en las zonas rurales de Guanacaste, especialmente, en los cantones de Santa Cruz y Liberia, iniciando las visitas al Bosque, con estudiantes de Educación General Básica, niveles de Educación Preescolar y Educación Primaria de instituciones aledañas al mismo.

El trabajo de aula abierta en las comunidades educativas propicia, además, la investigación científica de forma permanente, para monitorear los cambios del comportamiento humano ante la responsabilidad ambiental.

Actualmente, el Aula abierta se ha desarrollado como plan piloto; sin embargo, los resultados, según la experiencia de las visitas realizadas al lugar, principalmente por docentes y estudiantes de primaria y preescolar, reflejan satisfacción y entusiasmo, tanto en los recorridos, como a través de las experiencias didácticas dirigidas en el aula.

Es importante resaltar que, como parte de la formación de valores y actitudes positivas hacia los espacios naturales, se inserta, también, en el desarrollo del proyecto, a estudiantes avanzados de las carreras de Turismo Ecológico, Educación Preescolar y Educación Primaria , quienes participan en la realización de las distintas actividades con la supervisión de docentes universitarios especializados en Biología y Educación.

Las comunidades educativas que han participado en este plan piloto son las del Centro Infantil de la Universidad de Costa Rica en Liberia, el Centro Infantil y la Escuela de la Universidad de Costa Rica en Santa Cruz, la Escuela de Barrio Limón en Santa Cruz, la Escuela de Guardia, la Escuela de Moracia, la Escuela la Victoria, la Escuela Laboratorio John F. Kennedy, la Escuela El Capulín, la Escuela Aplicación Alba Ocampo y la Escuela Ascensión Esquivel. 
En el caso de las seis primeras comunidades, se desarrolló el Aula abierta que combina las actividades dentro de los propios centros educativos, con actividades en el Bosque Ramón Álvarez. En este último se realizaron caminatas de historia natural, donde los participantes tuvieron la oportunidad de involucrarse con diversos aspectos ecológicos. Como en este plan piloto se trabaja con niños de preescolar y de primer ciclo de Educación Diversificada, es fundamental dar instrucciones precisas antes de iniciar el recorrido, lo cual se hace en el corredor de las instalaciones del Bosque: ahí se facilita el equipo necesario para la seguridad de los niños, como capas y botas de hule; además se brinda una lupa a cada niño para que puedan explorar mejor su alrededor. Los niños ingresan al sendero acompañados de alguno de sus padres o encargados, de sus maestros y de los guías.

Como parte de las actividades que ya en el Bosque se realizan con los visitantes, cabe destacar las siguientes: en la entrada al sendero, se reflexiona sobre la importancia de los bosques y el por qué conservarlos; durante el recorrido se estudian relaciones entre los organismos que componen el ambiente, utilizando, por ejemplo, las plantas de ojoche que presentan agallas (Fig. 2), la relación de los espaveles, ceibos y otras especies de árboles, con los congos, murciélagos y aves que utilizan sus flores y frutos para alimentarse; se estudia la dinámica de los bosques conversando sobre la caída de ramas y árboles y los procesos de germinación relacionados con la regeneración, el papel de los hongos en los procesos de descomposición de la materia y el ciclo de nutrientes. También, se aprovecha la presencia de árboles con gambas, para conversar sobre la estructura de las plantas y su función.

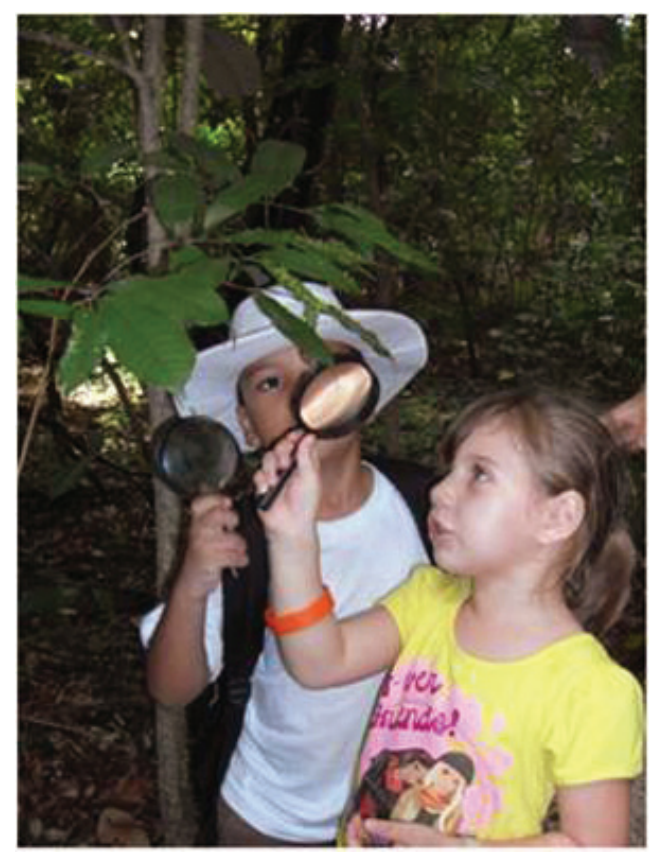

Figura 2. Estudiantes observando las agallas de una planta de ojoche, durante el recorrido por el sendero Bejuco.

Los participantes y las participantes que han vivido la experiencia en esta aula abierta comprenden aproximadamente 400 discentes de preescolar y primer ciclo de primaria, 28 docentes y 1200 miembros de familia, que participan de forma indirecta. 
En complemento a estas actividades, un equipo de docentes y estudiantes de la Carrera de Turismo Ecológico de la Universidad de Costa Rica, ha realizado monitoreo de flora y fauna, importantes como fuente de información biológica del lugar, para ser utilizada como referencia en los recorridos.

En las restantes comunidades educativas, el aula abierta orientó sus actividades al segundo ciclo de Educación General Básica. El tema de la cultura ambiental fue abordado a través de las Artes Plásticas como recurso pedagógico, partiendo de sus contextos y experiencias cotidianas, así como sus conocimientos y vivencias previas. Los temas se desarrollaron de forma lúdica, iniciando con la reflexión de diversos conceptos como cultura, patrimonio cultural, cultura ambiental, ecosistema, impacto ambiental, conservación, transformación, ética, reciclaje, áreas silvestres, recursos forestales e hídricos y biodiversidad, para posteriormente discutir los principales problemas ambientales presentes en la comunidad. Finalmente, se trataron de plasmar en proyectos artísticos, de una forma participativa, las principales ideas o intereses desarrollados en los discentes a través de estas actividades, utilizando en algunos casos, materiales de desecho.

\section{Resultados de la experiencia}

Estas actividades, utilizando como estrategia metodológica el uso de aula abierta, facilitaron no solamente la comprensión de aspectos relacionados con la naturaleza, sino también, han promovido mayor conciencia acerca del impacto que, como miembros del ecosistema, generamos a través de nuestras acciones cotidianas.

Las docentes y los docentes involucrados en el proyecto "Aula abierta" en las primeras seis escuelas, han manifestado que los niños y niñas han podido incorporar, como parte de su cultura ambiental, algunas actitudes importantes y manifiestan que tienen una mejor conciencia crítica de lo que consideran como actitudes inadecuadas hacia el medio. Las apreciaciones docentes son un punto valioso a considerar para el futuro fortalecimiento de la experiencia con el Aula abierta, así como las opiniones del estudiantado, padres y madres de familia y miembros de la comunidad en general.

A continuación, se presentan algunas observaciones hechas por las docentes y los docentes, en relación con algunas de las expresiones que es frecuente escuchar en quienes han participado activamente, tanto de las actividades dentro de los centros educativos, como en el Bosque Ramón Álvarez:

- "Cuando realizamos merienda en espacios exteriores manifiestan preocupación por la basura que no es depositada en el lugar indicado".

- "Cuando hacemos recorridos por la universidad comentan "los estudiantes de la U no saben utilizar los basureros, la tiran en el suelo, nosotros si cuidamos el planeta".

- "Cuando se cepillan los dientes utilizan un 'jarrito' para no dejar el tubo abierto y ahorrar el agua".

- "En Santa Cruz, un niño o niña está pendiente de que no dejen llaves del tubo abiertas".

- Por parte de los padres comentan: “QQué le dijeron a mi hijo? que solo vive hablando de cuidar el agua y no dejar basura tirada".

- "Posterior a la visita al Bosque, han tomado mayor interés para cuidar el ambiente, los árboles, la basura, ya no cortan las hojas a los árboles; quedaron encantados, fue muy lindo ir al Bosque, los hongos llamaron la atención, despertó curiosidad e interés por visitarlo nuevamente". 
El grupo de docentes considera de gran importancia iniciar, en esta etapa, la educación ambiental, porque los estudiantes y las estudiantes se convierten en un ejemplo para la familia y van creando consciencia, en sí mismos, para el futuro.

Al preguntar a las niñas y a los niños que visitaron el Bosque: ¿Por qué es importante el bosque, los árboles, el agua, el aire y otros elementos de la naturaleza?, entre otras expresiones, respondieron:

- "Nos limpian el aire".

- "No debemos cortar árboles porque el sol se pone más caliente, los árboles nos protegen del agua ".

- "Los árboles son importantes porque son la casa de los animales como capullos, mariposas, pájaros, búhos, hormigas: debemos cuidar la naturaleza, cuidemos el bosque".

Las personas involucradas en la ejecución de las actividades, después de realizar las caminatas de historia natural en el Bosque, han manifestado lo impactante que es, para las niñas y los niños, el vivir la experiencia de recorrer una zona natural, donde pueden estar en contacto directo con gran diversidad de plantas, hongos, insectos, sonidos, bejucos, semillas y olores del bosque; asimilan mucho mejor el porqué debemos cuidar nuestro medio.

Una vez finalizadas las caminatas por el Bosque, se realiza una plenaria con los niños y las niñas, donde se comentan y retoman algunos de los conceptos aprendidos en la clase de Ciencias. Los niños y las niñas son capaces de deducir, a partir de los conocimientos adquiridos durante las actividades lúdicas realizadas en los Centros Educativos, lo importante que es cuidar el medio ambiente.

Algunas de las manifestaciones expresadas por los niños y niñas, al finalizar los recorridos en el Bosque, relacionadas con contenidos del área de ciencias, fueron:

- "Las hojas tienen venas por donde toman agua, y el agua le da vida a la planta"

- "Cuando no hay plantas o árboles, se pone más caliente".

- "Los árboles son la casa de muchos animales como: aves, arañas, insectos, monos".

Las expresiones de los niños y las niñas denotan, claramente, el impacto que el contacto directo con las áreas naturales y su conocimiento puede provocar en la significatividad de los aprendizajes. En ese sentido, es mucho más claro enlazar los contenidos programáticos, por ejemplo, los referidos a educación ambiental, cuando los mismos son contextualizados mediante la experiencia del Aula abierta. Es ahí, justamente, donde dicho contenido cobra significatividad y, por tanto, promueve el aprendizaje y la modificación de comportamientos en contra de la naturaleza, así como el fortalecimiento de actitudes positivas hacia el ambiente en general.

Paralelo a las actividades de Aula abierta aquí expuestas, se desarrollaron actividades en el campo artístico-ambiental, con discentes de segundo ciclo de Educación General Básica, a cargo de una profesional en dicho campo. Los resultados obtenidos de estas experiencias muestran que los discentes desconocen conceptos y confunden términos como: cultura ambiental, ecosistemas, impacto ambiental, entre otros. Podría deducirse que estos niños y niñas presentan desconocimiento acerca de las problemáticas ambientales tratadas durante las actividades, por lo que los conceptos quedan en un nivel abstracto.

Como parte de la estrategia para hacer más comprensibles los aspectos estudiados, se han incorporado, en las discusiones de los temas citados, los problemas ambientales de sus comunidades, 
lo cual ha facilitado y promovido una mejor comprensión, por parte de los discentes, de los aspectos abarcados.

Al incluir en la experiencia temas que los involucran como actores, tales como la contaminación ambiental y la producción de desechos, el estudiantado ha mostrado una disociación entre la conciencia ambiental que posee y lo que vive diariamente en su comunidad, ya que, a pesar de ser conscientes de la necesidad de cuidar el ambiente, no actúa en forma acorde con estos principios.

De igual manera, se ha podido observar, en algunos de los centros educativos visitados, material de desecho acumulado, basura en el piso, lo que evidencia descuido por el ornato, manifestando que esa no es una labor propia de la institución. Por otra parte, los niños y niñas están conscientes de las actividades y conductas seguidas por los miembros del núcleo familiar, que muestran incongruencia con la conservación del ambiente.

En relación con algunos problemas ambientales, como contaminación por gases, quemas, el mal manejo de aguas negras y jabonosas, los grupos de discentes manejan información de manera poco profunda, y manifiestan frustración, pues consideran que son aspectos en los cuales no tienen participación, ya que son las acciones de los mayores las que fomentan el deterioro ambiental.

Las actividades lúdicas de esta Aula abierta tratan de hacer comprender a sus participantes, que cada quien es capaz de ejecutar acciones y cambiar hábitos que favorezcan la conservación del medio ambiente circundante. La confección de murales en forma conjunta, con referencia a algún tema ambiental, pretende favorecer procesos de concientización ambiental, que vayan de la mano con un crecimiento de la creatividad; libertad de expresión; capacidad crítica y solidaria, hacia ellos mismos, los demás y su ambiente. Esto favorece un cambio positivo en la cultura ambiental de los discentes y repercute en una sociedad futura practicante de un verdadero desarrollo sustentable (Alvarado, 2010).
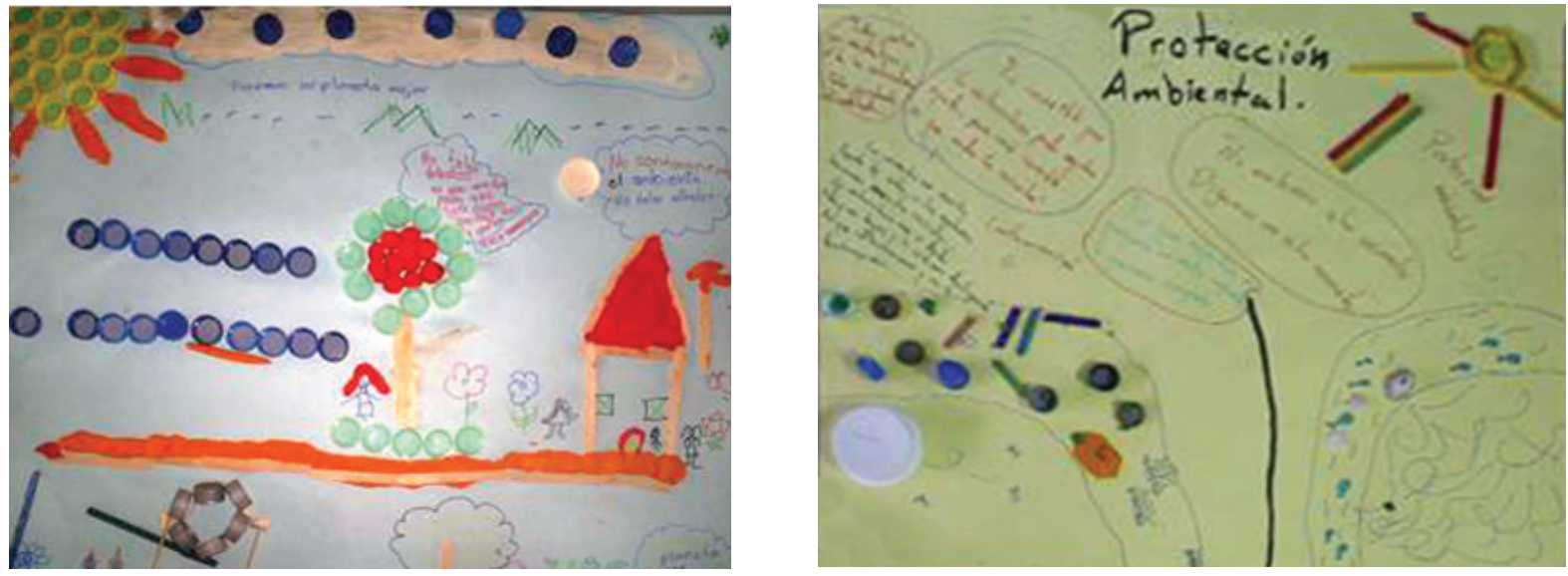

Figura 3. Murales realizados por los discentes de la Escuela Aplicación Alba Ocampo (a) y la Escuela Laboratorio John F. Kennedy (b). En la primera de ellas, se argumenta la necesidad de construir un planeta mejor para todos, mientras que en la segunda se hace un manifiesto de la necesidad de un cambio radical, a través de frases como: "Es increíble como la naturaleza pide a gritos ayuda, pero más increíble es que nadie la escuche.", “Todos juntos por nuestro bien y por el de la naturaleza", "Los animales son nuestra fuente de vida, la naturaleza nos ha dado vida, un hogar y la estamos destruyendo..."

\section{Conclusiones}

El plan piloto de Aula abierta, que combina actividades lúdicas dentro de los Centros Educativos, con caminatas de historia natural en el Bosque Ramón Álvarez, parece ser una excelente opción, pues permite plasmar mejor los conceptos biológicos y crear una verdadera conciencia ambiental. 
Es evidente, según las valoraciones de los docentes y las docentes, así como las respuestas estudiantiles, un cambio de actitud por parte de los niños y niñas participantes en las actividades. Después de la experiencia parecen más conscientes de sus actos y de los actos de los demás, en cuanto al impacto que tienen sobre el medio, como es el caso del uso del agua.

Es importante que los remanentes de Bosque, que aún quedan en nuestra deforestada Guanacaste, puedan ser usados productivamente, no como fuente de madera, plantas ornamentales, sino como verdaderas aulas, donde los visitantes aprendan sobre el funcionamiento de los ecosistemas y lleguen a considerarse parte del mismo, para que se conviertan en verdaderos abogados ambientalistas.

De igual manera, es importante motivar a los cuerpos docentes se la región, para que hagan uso de las áreas silvestres cercanas a sus centros educativos, en pro de una mejor comprensión del ambiente natural y que, realmente, desarrollen discentes capaces de comprender que constituimos parte de un gran ecosistema y que nuestros actos impactan directamente en él. Para ello, es necesario el uso de estrategias de aprendizaje y metodologías participativas, reales y contextualizadas, que permitan la flexibilización curricular verdadera, que integre a los diferentes actores sociales y, principalmente, a niños y niñas.

Vale la pena señalar, también, que la experiencia piloto desarrollada en el Bosque Ramón Álvarez en Guanacaste constituye una estrategia viable de desarrollar en otras comunidades del país, en donde haya disponibilidad de zonas naturales o protegidas que puedan constituirse en laboratorios permanentes para los niños y las niñas costarricenses y, a la vez, se conviertan en recursos disponibles para sus docentes.

Es necesario resaltar que cuando el trabajo con contenidos específicos se enlaza con la cotidianidad y se contextualizan en la propia naturaleza, no solo adquiere significatividad para el estudiantado, sino que, además, favorece la promoción y formación de valoración ambientalistas y conservacionistas, que permitan, a las generaciones futuras, disfrutar y cuidar el planeta, lo cual es una responsabilidad de todos y todas.

Invitamos entonces, a los docentes y a las docentes, a incorporarse a experiencias de aula abierta en espacios naturales ya brindar, de esta manera, a sus estudiantes, la oportunidad de conocer, disfrutar, valorar, respetar y cuidar su entorno natural.

De igual manera, instamos a los miembros de las distintas comunidades a identificar las áreas por conservar, a incorporarse activamente en su defensa y preservación, y a reforzar, en la población, los valores que se promueven en las instituciones educativas para la defensa y conservación de la naturaleza.

\section{Referencias bibliográficas}

Alvarado, R. (2010). Cultura ambiental en acción (Informe parcial). Región Chorotega, C. R.: CONARE.

Baltodano, J. (2008). Bosque coberturas y uso forestal (Décimocuarto informe estado de la nación en desarrollo humano sostenible). Recuperado de http://www.coecoceiba.org/wp.../11/ Bosques-coberturas-Baltodano-2008.pdf

Barrantes, G. (2000). Aplicación de incentivos a la conservación de la biodiversidad en Costa Rica. San José, Costa Rica: Sistema Nacional de Áreas de Conservación, MINAE. 
Calderón, M. (2005, Agosto). El turismo como promotor del crecimiento económico costarricense. Revista Parlamentaria Digital, 13(2), 263-270.

Castro, R., Monge, E., Rocha, C. y Rodríguez, H. (2004). Gestión local y participativa del recurso hídrico en Costa Rica. San Jose, Costa Rica: Centro de Derecho Ambiental y de los Recursos Naturales (CEDARENA). Programa Gestión Integrada del Recurso Hídrico. Recuperado de http://hidrico.sociedadhumana.com/media files/download/gestion.pdf

Coen, E. (1991). Clima. En D. Janzen (Ed.), Historia natural de Costa Rica (pp. 35-46). San José, Costa Rica: Editorial Universidad de Costa Rica.

Davis, R. y Holmgren, P. (2000). FRA 2000. Bibliografía comentada. Cambios en la cobertura forestal Costa Rica. Roma: Programa de Evaluación de los Recursos Forestales. Organización de las Naciones Unidas para la Agricultura y la Alimentación. Recuperado de http://www.fao. org/docrep/007/ad668s/ad668s00.HTM

Ewel, J. J. (1999). Natural Systems as Models for the Design of Sustainable Systems of Land Use [Los sistemas naturales como modelos para el diseño de sistemas sostenibles de uso de la tierra]. Agroforestry Systems 45(1-3), 1-21. doi: 10.1023/A:1006219721151

García, R. (2002). Biología de la conservación: conceptos y prácticas. Heredia, Costa Rica: Editorial INBio.

Gutiérrez, O. (2006, octubre). Flexibilidad curricular. (Taller: Diversificar las experiencias de formación profesional [Presentación Power Point]). Recuperado de http://www.anfei.org.mx/ $\underline{\text { XI RGD/Taller Flexibilidad Ofelia Angeles.pdf }}$

Hartshorn, G. S. (1991). Plantas. En D. Janzen (Ed.), Historia natural de Costa Rica (pp. 119-185). San José, Costa Rica: Editorial Universidad de Costa Rica.

Hernández, E. y Zúnica, I. (2005). La cuenca hidrográfica como unidad alternativa para el desarrollo turístico. El caso de la cuenca del Río Morote. Guanacaste. Costa Rica. Ponencia presentada en el Seminario Regional "Turismo Alternativo en la región centroamericana: Experiencias y acciones conjuntas para un desarrollo local de las comunidades". Nicoya, Costa Rica. Recuperado de http://files.riomorote.webnode.es/200000222-a7bb6a8b55/PONENCIA\%20 CUENCA\%20MOROTE-PUBLICACI\%C3\%93N Igor\%20Zu\%C3\%Bliga 2006.doc

Leenders, T. (2001). A guide to Amphibians and Reptiles of Costa Rica [Una guía de anfibios y reptiles de Costa Rica]. Florida: Distribuidores Zona Tropical S. A.

Merayo, O. (2004). Valoración económica del agua potable en la cuenca del río Endemedio, Santa Cruz, Guanacaste, Costa Rica. Recursos Naturales y Ambiente, 43, 90-96. Recuperado http:// orton.catie.ac.cr/repdoc/A3190E/A3190E.PDF

Meza, T. A. (2001). Geografía de Costa Rica: geología, naturaleza y políticas ambientales. Cartago, Costa Rica: Editorial Tecnológica de Costa Rica. 
Moreno, M. L. (2005). Pago por servicios ambientales, la experiencia de Costa Rica. Costa Rica, s. e. Recuprado de http://www.inbio.cr/otus/pdf/informe-servicios-ambientales.pdf

Quesada, G. (2008). Garantías ambientales: un nuevo modelo ecológico-político para Costa Rica y el mundo. Biocenosis, 21(1-2), 119-124.

Ramírez, E. (miércoles 14 de abril de 2010). Recursos hídricos y bosques afectados: persisten daños ambientales en el país y amenazan con crecer. Semanario Universidad. Recuperado de http://www.semanario.ucr.ac.cr/ index.php/mainmenu-pais/2180-recursos-hidricos-y-bosquesafectados-persisten-danos-ambientales-en-el-pais-y-amenazan-con-crecer-html

Sánchez-Azofeifa, A., Kalácska, M., Quesada, M., Stoner, K., Lobo, J. A. y Arroyo-Mora, J. P. (2003). Tropical Dry Climates [Los climas tropicales secos]. En M. D. Schwartz (Ed.), Phenology: An Integrative Environmental Science [Fenología: una ciencia ambiental integrada] (pp. 121137). Estados Unidos: Kluwer Academic Press.

Valerio, C. E. (1991). La diversidad biológica de Costa Rica. San José, Costa Rica: Editorial Heliconia.

Wainwright, M. (2002). The Natural History of Costa Rican Mammals [La historia natural de los mamíferos de Costa Rica]. Florida: Zona Tropical.

Zamora, N., Jiménez, Q. y Poveda, L. J. (2002). Árboles de Costa Rica. Trees of Costa Rica (Vol II). Heredia, Costa Rica: Editorial INBio.

Zuchowski, W. (2005). A guide to Tropical Plants of Costa Rica [Una guía para las plantas tropicales de Costa Rica]. Florida: Zona Tropical. 